\title{
Ginginerering Jourtulal
}

Article

\section{Tire-Suspension-Steering Hardware-in-the-Loop Simulation}

\author{
Sunhapos Chantranuwathana ${ }^{1,2, *}$, Ratchatin Chancharoen ${ }^{1}$, Witaya Wannasuphoprasit ${ }^{1}$, \\ Angkee Sripakagorn ${ }^{1,2}$, and Nuksit Noomwongs ${ }^{1,2}$
}

1 Department of Mechanical Engineering, Faculty of Engineering, Chulalongkorn University, Bangkok 10330, Thailand

2 Smart Mobility Research Center, Chulalongkorn University, Bangkok 10330, Thailand

*E-mail: supavut@gmail.com (Corresponding author)

\begin{abstract}
Safety and performance have always been important factors in automotive testing. These factors are highly dependent on the tires and suspensions, which should be simulated and tested throughout the development process. During development, Hardware-In-the-Loop (HIL) simulations may be used so that testings and tunings can be done earlier in the process. In this paper, designs and configurations of a newly developed tire-suspension-steering HIL are shown. An actual wheel assembly with suspension and steering components can be installed for testing with dynamic models of the rest of the car. The slip angle of the tire can be imposed in the test rig while actual tire forces can be measured and used in the dynamic model. Comparisons of HIL simulations and real experiments using the skidpad test and the step steering test are given using Formula SAE race cars. It was found that the HIL simulation results are more accurate compared to non-HIL simulations.
\end{abstract}

Keywords: Vehicle dynamics, tire suspension steering hardware in the loop simulations, skidpad test, step steering response.

ENGINEERING JOURNAL Volume 22 Issue 5

Received 30 January 2018

Accepted 26 July 2018

Published 30 September

Online at http://www.engj.org/

DOI:10.4186/ej.2018.22.5.199 


\section{Introduction}

Safety and performance have always been important factors in automotive dynamic testing. These factors are highly dependent on the tires and suspensions [1-3] which should be simulated and tested throughout the development process. Recently, HIL simulations have become increasingly common. With HIL simulations, highly reproducible testings and tunings can be done earlier in the design and validation process. Applications of HIL simulations can be found in various stages of the so-called "V" diagram of the design and development process [1]. In this paper, a newly developed tire, suspension, and steering HIL test rig is presented.

An HIL simulation is a testing method that combines advantages of hardware based and model based testing $[3,4]$. In such simulation, real components that need to be tested or that are difficult to build mathematical models for are used, while mathematical models are used to simulate other parts of the system that may not exist at the time of testing. Generally, HIL simulations can reduce cost and testing time when compared to a full prototype testing. In some cases, HIL simulations can also be used to bypass the need to experimentally construct a complicated mathematical model.

In automotive applications, one of the most common applications of HIL simulations is testing and evaluating a vehicle's Electronic Control Unit (ECU). In this case, a real ECU is tested by giving it virtual signals generated from a computer running a vehicle model. It can then be tested and analyzed under various driving conditions [5]. Dangerous driving conditions can be tested safely and repeatably. Due to these advantages, there are a number of commercially available products specialized in HIL simulations of ECUs; e.g., products from AVL, dSPACE, and ETAS. There are numerous HIL systems to test various functions of the ECU; e.g., HIL of Anti-lock Brake Systems (ABS) [6,7], HIL of Electronic Power Steering (EPS) [8], HIL for testing of velocity/distance controller for driver safety system [9], HIL system of throttle body for testing of engine throttle controller [10], and HIL system to test diesel hybrid electric vehicle [11].

Apart from the ECU, there are many other HIL systems developed for testing of other automotive systems. The following are some of the HIL systems that may be related to the proposed tire-suspensionsteering system. For example, a wheel and tire HIL system for testing of vehicle dynamics was developed in [12] and [13]. Damper and suspension HIL systems were developed to study vertical vibration [3,14]. Steering HIL systems can also be found in the works by [8] and [15]. A combination of brake, steering, and tire pressure HIL system was presented in [16]. There was even a system with a full car in the simulation loop to test driver assistant systems [1].

In terms of vehicle dynamic testings, which are the main focus of this work, there are a number of HIL systems that can be found in the literature. However, these are mostly HIL of separate components. The HIL system with a combination of tire, suspension, and steering components developed in this work has not been used before. Arguably, the force generation between the tire and the road is the most important and the most complicated. Hence, an HIL system with actual tires were proposed in $[12,13]$. An HIL system was constructed with two go-kart tires to measure actual tire lateral forces and used in a single track vehicle model. There were neither suspension nor steering components but the tires can be positioned to impose the slip angle required by the model. Motion and deformation of the suspension and steering components cannot be simulated by this hardware. An HIL system of vehicle suspension dampers [17] and an HIL system of other suspension components [3] were also studied. However, the vertical vibration was the main focus. Similarly, steering HIL systems were used to study steering dynamics $[8,18]$. Actual steering components were used while the tire forces were simulated. It should be noted that there are a number of studies that use scaled vehicles that include the tire, the suspension, and the steering system $[19,20]$. However, the overall dynamic behaviors of the car were the main concerns. The suspension and steering components of the scaled vehicle were not meant to replicate the actual components.

In this paper, a newly developed tire, suspension, and steering HIL test rig is presented. In this system, actual tire, suspension, and steering components can be installed and used with a mathematical model of the rest of the car. In the literature, only a few HIL systems have focused on capturing 
lateral tire forces with actual tires [12, 13, 21, 22]. Capturing accurate lateral tire forces is important when simulating vehicle handling tests; e.g., skidpad tests and step steering tests. In the literature, the suspension and steering components were used in HIL systems separately. However, the accuracy of such tests may be improved if the components are tested together, since tire, suspension, and steering components interact with each other. For example, the lateral tire force generates deflection in the tire as well as in the suspension and the steering components. To capture their interactions, an HIL test rig that combines tire, suspension, and steering components is proposed in this work.

Comparisons of HIL simulation results with real vehicle experiments using Formula SAE race cars are also presented in this paper. To study the lateral dynamic behavior of a car, a skidpad test and a step steering test are usually performed [12,13,23,24]. However, only a few studies have published data comparing HIL results with full car experiments. For example, an HIL system was constructed with a pneumatic brake and an ECU was used to simulate truck dynamics to test stability controllers [25]. Although experimental data was available, HIL results were not directly compared to the experimental data. The work focused on the improvement of the control system predicted by the HIL results.

\section{Design and Construction}

In this HIL system, actual vehicle wheel assemblies with tire, suspension, and steering components can be installed as shown in Fig. 1. The system was designed to allow various types of suspensions to be installed. As shown in the figures, a McPherson strut (Fig. 1a), a tricycle front fork (Fig. 1b), and a double wishbone suspension (Fig. 1c) can be installed. Furthermore, a steering motor and a driving motor can also be installed to study the front wheel or the rear wheel as desired. For the front wheel, it can be steered to follow a simulated driver command. The wheel is placed on top of a road surface drum that can be oriented to create the slip angle of the tire while the resulting tire force is measured.

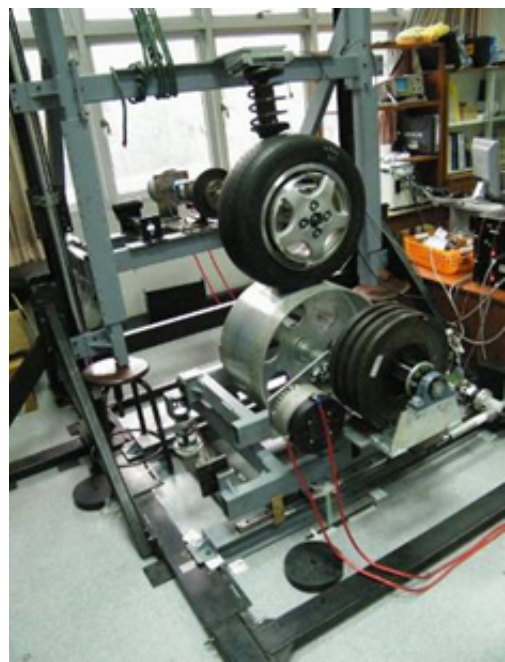

(a) MacPherson strut

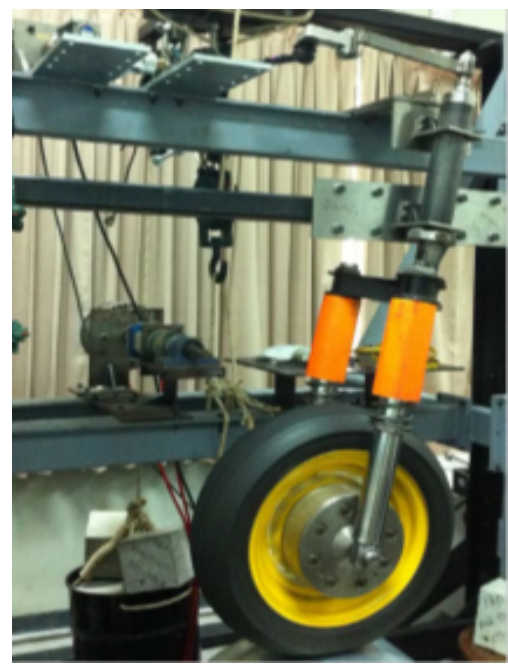

(b) Front fork of a tricycle

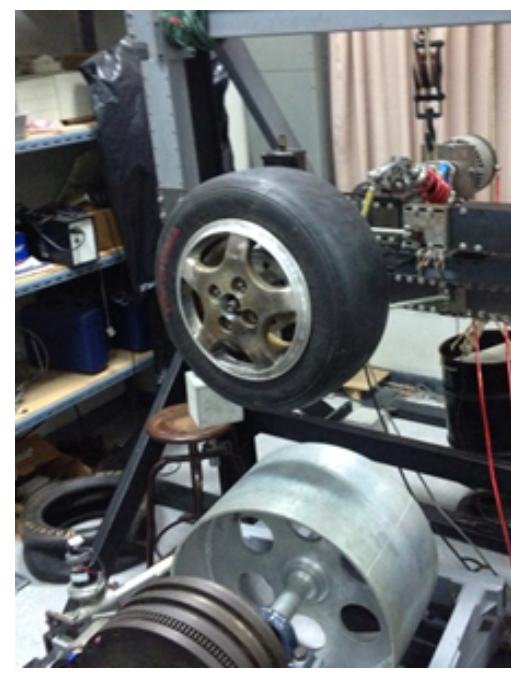

(c) Double wishbone

Fig. 1. Tire-Suspension-Steering HIL test rig.

The road surface drum, which is a key feature of this HIL test rig, is shown in Fig. 2. The drum, which is $600 \mathrm{~mm}$ in diameter, was used to simulate the road surface. The speed of the drum can be controlled to match the speed of a simulated car. An equivalent vehicle speed of approximately 25 $\mathrm{km} / \mathrm{h}$ can be simulated with the installed motor. The car mass can also be simulated with rotational inertia of the road surface drum which can be adjusted with inertia disks. A timing belt was used to speed up the inertia disks, resulting in lighter inertia disks as shown in the figure. It is important to note that the slip angle of the tire is not the same as the steering angle. To impose the slip angle, the drum system was designed so that it can be oriented relative to the wheel. Furthermore, since the tire 
contact patch will move when the wheel is steered, the drum system can also be positioned to keep the tire contact patch at the center of the wheel. Two linear actuators shown in the figure were used to control the position and orientation of the road surface drum. The drum system can be moved linearly for $\pm 100 \mathrm{~mm}$ and can be rotated for $\pm 25^{\circ}$.

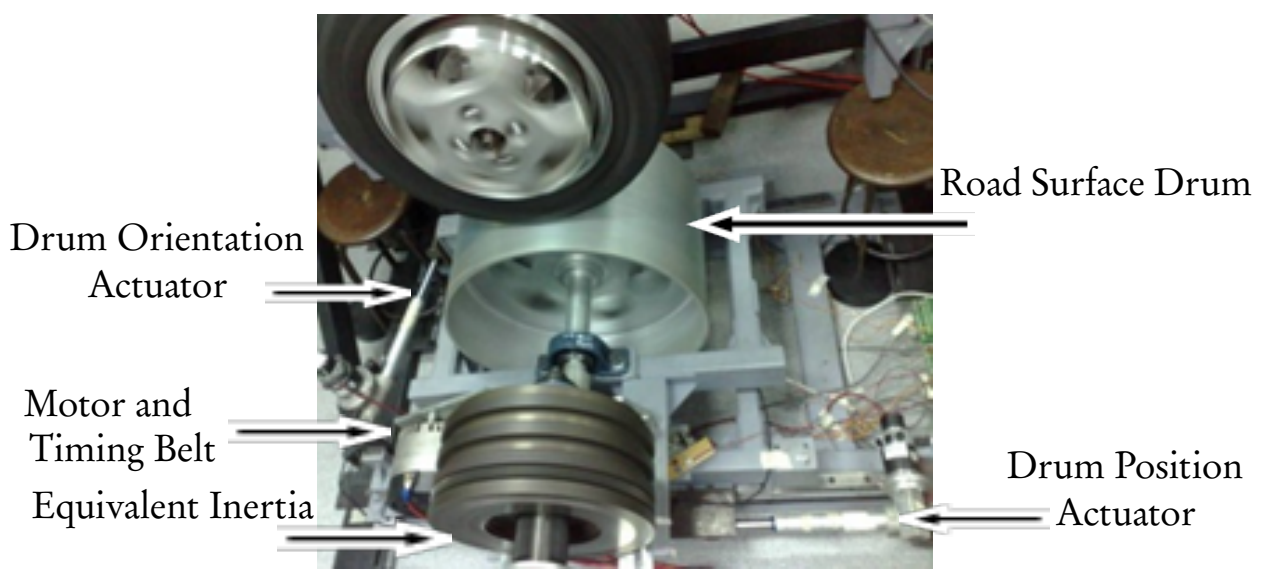

Fig. 2. Road surface drum and its actuators.

One of the main difficulties in building this HIL system was the tire force measurement system. For this purpose, a rotating force measuring device in a form of a wheel rim is generally used. This type of device is, however, expensive. To reduce cost, a ring-shaped non-rotating force transducer was used. Because of the size of this force measuring device, it is impossible to use the same knuckle as the real formula car. The knuckle must be modified to install the force transducer in the middle of the knuckle as shown in Fig. 3. Notice that a special rim with a large positive offset must be used to compensate for the thickness of the force sensor. This is to ensure that the tire contact patch remains at the original location relative to the suspension. Furthermore, the brake system must also be moved inbound to accommodate for the increased in size of the wheel hub assembly as shown in Fig. 4. The chosen ring-shaped transducer also allows a drive shaft to pass through to drive the wheel if required. For other types of cars or suspensions, similar modifications may also be applied. For example, Fig. 5 shows a modified front wheel assembly of a tricycle. The force sensor was secured on one side of the main shaft which ran through the force sensor. Bearings were installed on the other side of the sensor so that the wheel can freely rotate on them.

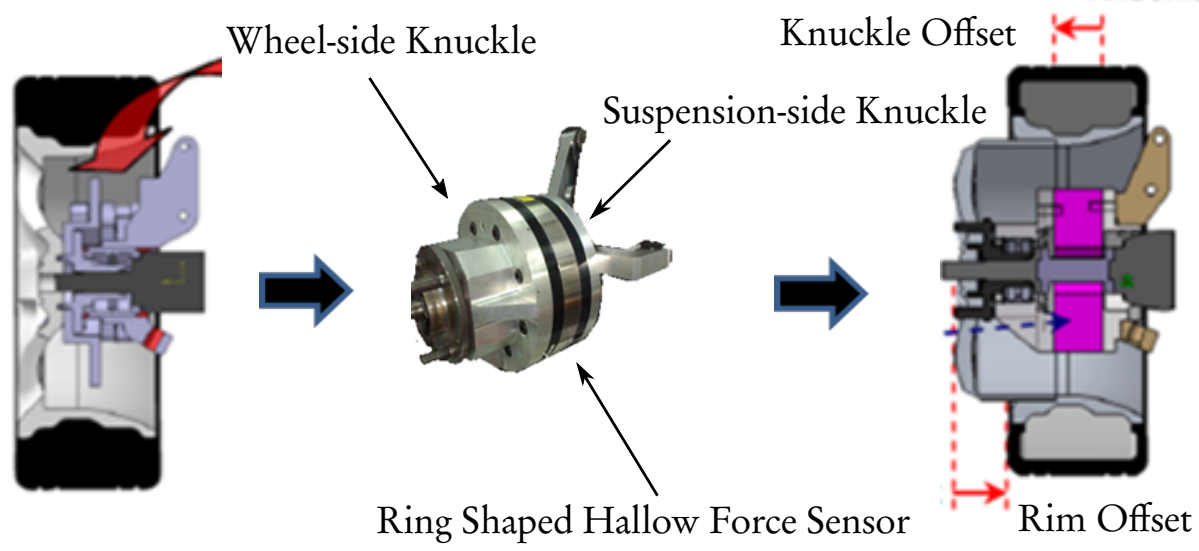

Fig. 3. Wheel assembly of a Formula SAE car with the knuckle modified to include the force sensor.

To simulate a Formula SAE car, a double wishbone suspension of the car was installed. Mounting 


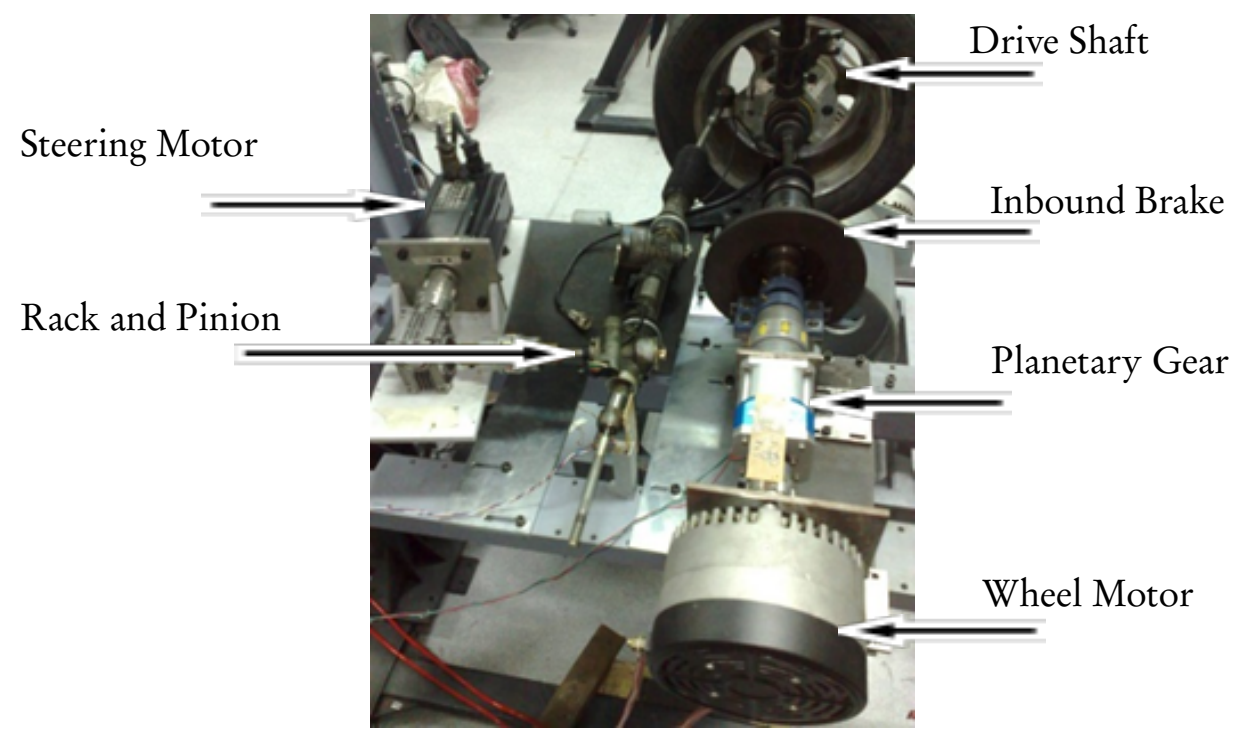

Fig. 4. Steering and driving system.

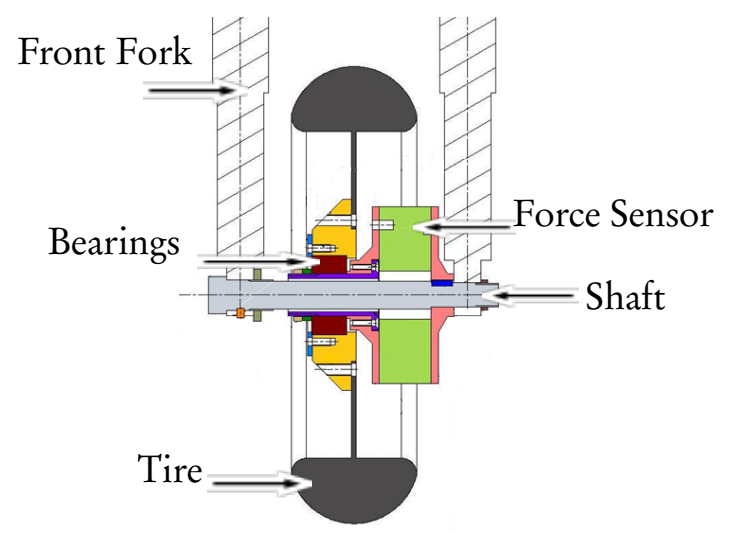

Fig. 5. Front wheel assembly of a tricycle.

points for the suspension, spring, and damper were replicated in the exact locations as in the real Formula SAE car. The bell crank, the push rod for the spring, and the damper were also duplicated. Two versions of the Formula SAE car were implemented and simulated. Both versions used slick tires with 13 inches rim and 7.5 inches of width (180/50V R13). The effective diameter of the installed tire was approximately $540 \mathrm{~mm}$ at 25 psi of inflation pressure. Figure 6 shows the actual suspension on the HIL test rig. The wheel assembly, the suspension components, and the steering components were installed on a carriage mounted on two vertical guide rails. Vertical load on the tire can be adjusted by adding or removing masses from the carriage.

The overall interactions between the simulation software with the mathematical model of the car and the tire-suspension-steering hardware are shown in Fig. 7. In an HIL simulation where the actual front wheel is used, the steering command and the driving torque (or the vehicle speed) are the inputs to the car model depending on the driving scenario. The tire slip angle, the wheel speed, and the vehicle speed, which can be obtained from the model along with the steering angle inputs, are used as the reference input for the low-level hardware controllers. For example, the wheel steering angle is controlled using a rack and pinion steering system by a low-level PID controller to follow the steering reference input. The orientation of the road surface drum is controlled relative to the wheel to follow the reference tire slip angle. The tire lateral force is measured and used as another input to the car model. 

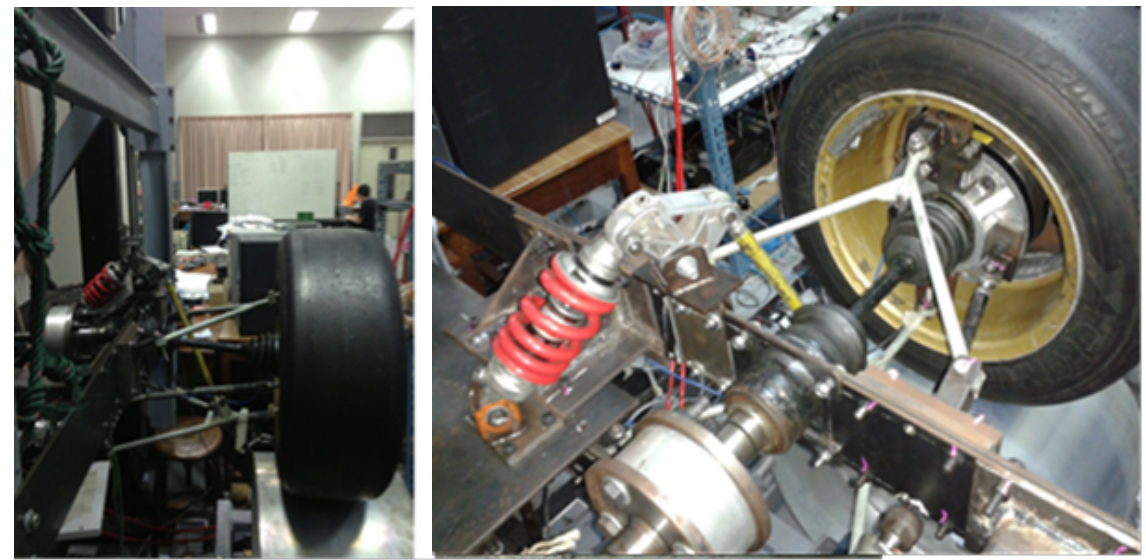

Fig. 6. HIL system with Formula SAE car components.

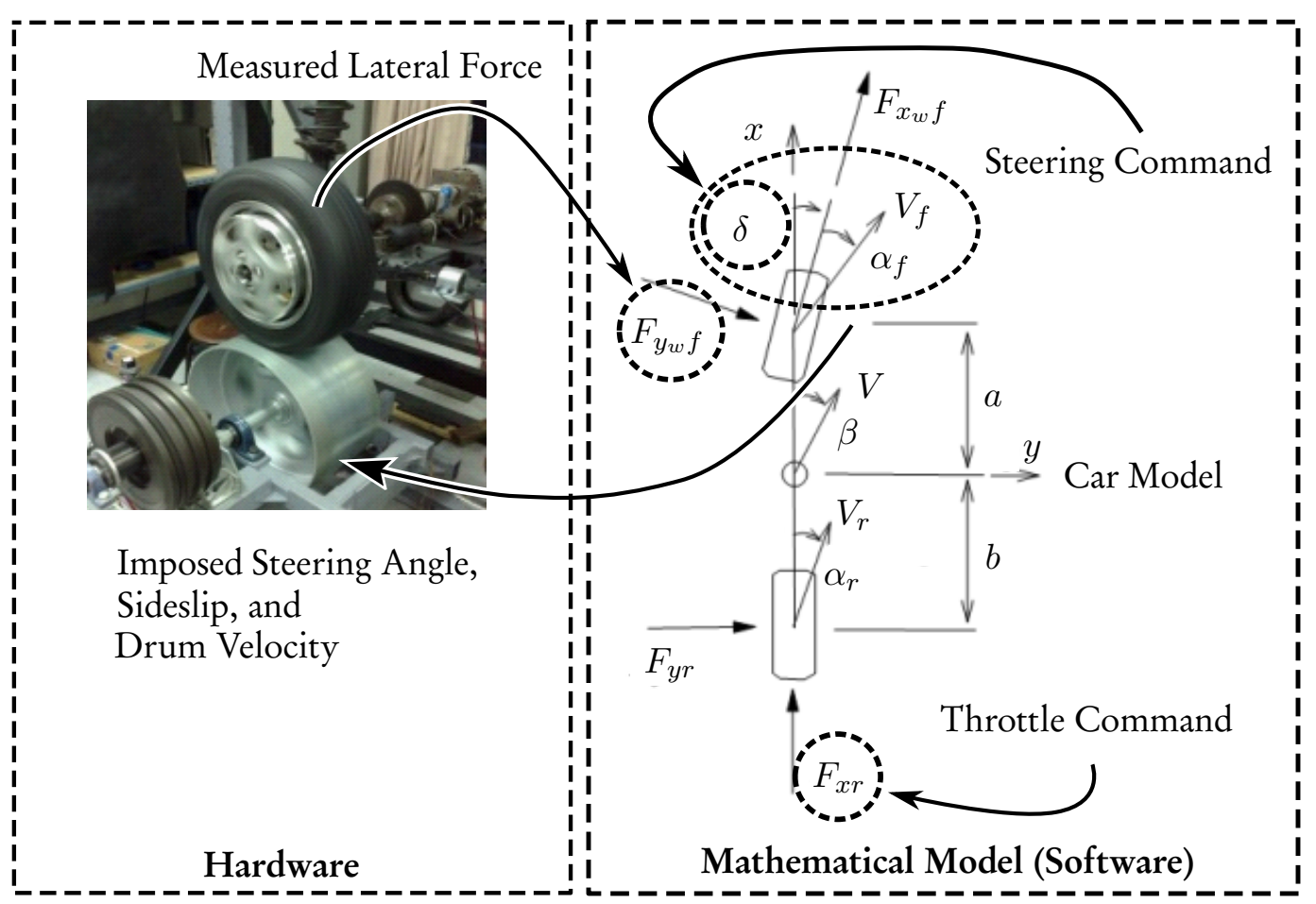

Fig. 7. Hardware-In-the-Loop simulation scheme.

Figure 8 shows the control system of the HIL test rig. To operate this system, a CompactRIO and a PXI based computer from National Instrument are used. The mathematical model of the car is simulated on the CompactRIO. Five low-level PID controllers are also implemented on the CompactRIO. The PID controllers are used to control the five actuators: the steering motor, the road surface drum orientation motor, the road surface drum position motor, the road surface drum rotation motor, and the tire speed motor, using the corresponding reference values from the model. The PXI computer is used to read from the force sensor and transmit the force values to the CompactRIO via a TCP/UDP network. 
Tire-Suspension-Steering Hardware

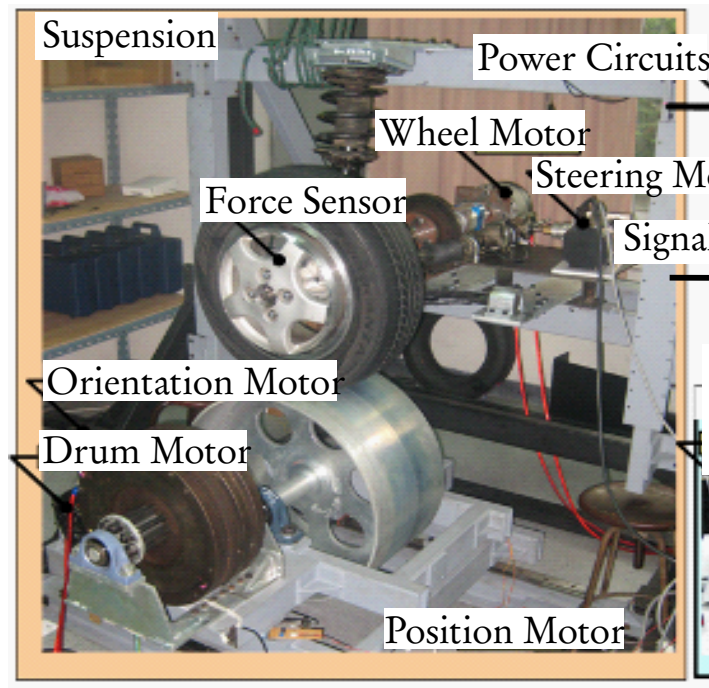

Fig. 8. HIL control systems.

\section{Power Circuit and Interface Box}

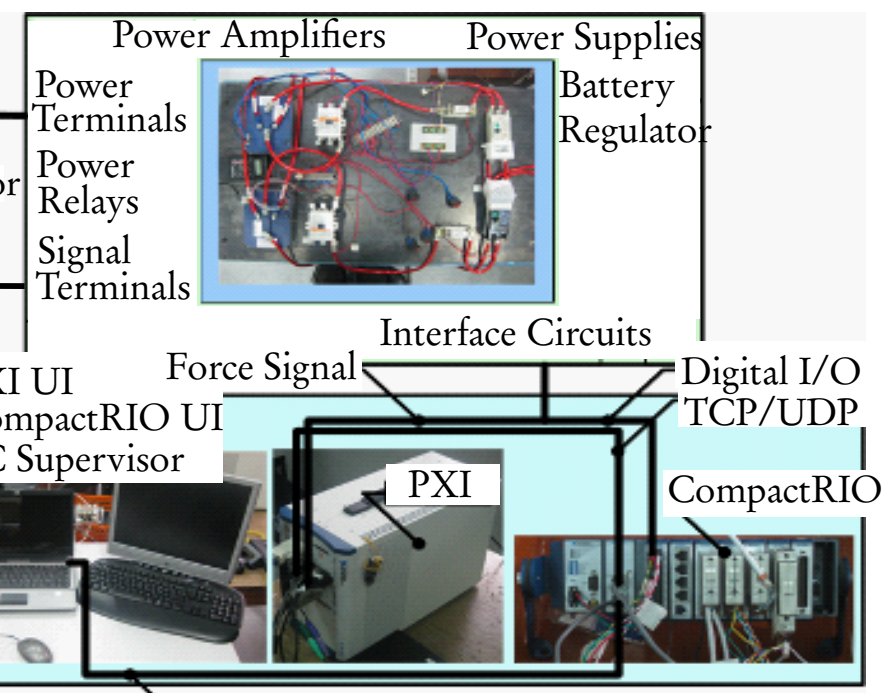

TCP/IP

Simulator and Low Level Controllers

\section{Vehicle Model}

The single track model or the so-called "bicycle" model used in this work is shown in Fig. 9. Inputs to the model are the wheel steering angle $(\delta)$, the two longitudinal forces $\left(F_{x_{w} f}\right.$ and $\left.F_{x r}\right)$, and the two tire lateral forces $\left(F_{y_{w} f}\right.$ and $\left.F_{y r}\right)$. As stated earlier, the first three inputs are dependent on the driving scenario. One of the two lateral forces is measured from the test rig while the other is obtained from a tire model. For example, if the front wheel and suspension system are installed on the test rig, then the front tire lateral force is measured while the rear lateral force is obtained from a model. The front slip angle $\left(\alpha_{f}\right)$ can be obtained from the model, which must be replicated on the HIL hardware. The state equations of the single track model can be written as

$$
\begin{aligned}
\dot{V}_{x} & =r V_{y}+\frac{1}{m}\left\{F_{x_{w} f} \cos \delta-F_{y_{w} f} \sin \delta+F_{x r}\right\} \\
\dot{V}_{y} & =-r V_{x}+\frac{1}{m}\left\{F_{x_{w} f} \sin \delta+F_{y_{w} f} \cos \delta+F_{y r}\right\} \\
\dot{r} & =\frac{1}{I_{z}}\left\{a F_{x_{w} f} \sin \delta+a F_{y_{w} f} \cos \delta-b F_{y r}\right\}
\end{aligned}
$$

where $V_{x}$ is the longitudinal speed; $V_{y}$ is the lateral speed; $r$ is the yaw rate; $m$ is the mass of the vehicle; $F_{x_{w} f}$ and $F_{y_{w} f}$ are the tire forces at the front wheel in the $x$ and $y$ directions relative to the wheel; $F_{x r}$ and $F_{y r}$ are the tire forces at the rear wheel in the $x$ and $y$ directions; $I_{z}$ is the mass moment of inertia of the car; $a$ and $b$ are the distances from the center of mass to the front and the rear wheels respectively; $\delta$ is the steering angle; $\beta=\arctan \left(V_{y} / V_{x}\right)$ is the vehicle's sideslip angle; and $\alpha_{f}$ and $\alpha_{r}$ are the tire slip angles of the front and rear tire respectively.

\subsection{Formula SAE Car}

The vehicle model has a number of parameters. In this case, some parameters are known while others have to be measured from the actual Formula SAE car. Table 1 shows the parameters of the Chulalongkorn 2012 Formula SAE car shown in Fig. 10. The mass, the distance $a$, and the distance $b$ can be measured directly without much difficulty. On the other hand, the mass moment of inertia is obtained 


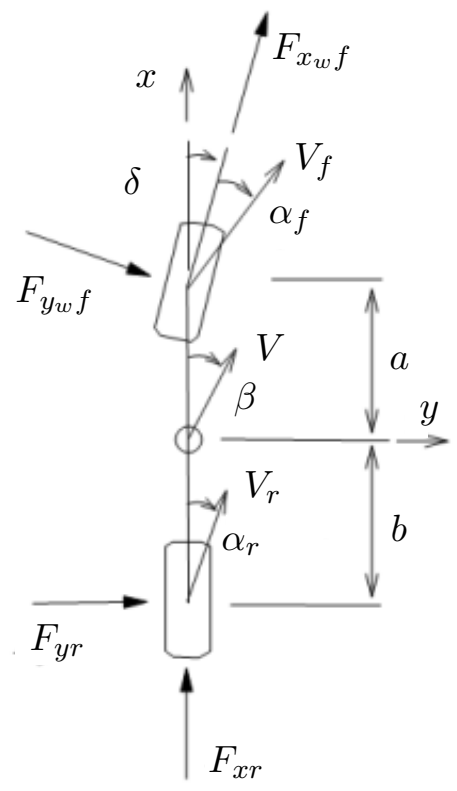

Fig. 9. Single track model.

by rotating the car about a vertical axis with a known rotational stiffness; i.e., a torsional pendulum. The simple harmonic motion obtained can be used to calculate the inertia.

Table 1. Formula SAE car parameters.

\begin{tabular}{|l|c|}
\hline Car mass $(\mathrm{m})$ & $366 \mathrm{~kg}$ \\
\hline Moment of inertia $\left(I_{z}\right)$ & $146.22 \mathrm{~kg} \cdot \mathrm{m}^{2}$ \\
\hline CG to front wheel $(\mathrm{a})$ & $0.839 \mathrm{~m}$ \\
\hline CG to rear wheel $(\mathrm{b})$ & $0.821 \mathrm{~m}$ \\
\hline
\end{tabular}

\subsection{Lateral Tire Force and Cornering Stiffness}

A tire force model is required to use the vehicle model. For a non-HIL simulation, models for both front and rear tires are required. For the HIL simulation in this work, only the rear tire model is required. The relation between the slip angle $(\alpha)$ and the lateral force $\left(F_{y}\right)$ was measured in advance by using the HIL test rig. For this purpose, the road surface drum was oriented at multiple angles to impose slip angles while the lateral forces were measured. Figure 11 shows the relationship between the lateral forces and the slip angles. In this plot, the front wheel vertical load was $90 \mathrm{~kg}(882.9 \mathrm{~N})$ and the rear wheel vertical load was $92 \mathrm{~kg}(902.5 \mathrm{~N})$ which are the static loads of the wheels. Various speeds of the drum were also used in the experiments.

Based on Fig. 11, linear relationships between lateral forces and slip angles of the tires are generally used to estimate understeer/oversteer behavior of a car. In particular, the center slope of the graph may be defined as the tire cornering stiffness: $C_{\alpha f}$ for the front tire and $C_{\alpha r}$ for the rear tire. From the graph, $C_{\alpha f}$ is $242 \mathrm{~N} / \mathrm{deg}$ and $C_{\alpha r}$ is $289 \mathrm{~N} / \mathrm{deg}$. Equation (2) can then be used to calculate the understeer gradient $(K)[24]$.

$$
K=\frac{w_{f}}{C_{\alpha f}}-\frac{w_{r}}{C_{\alpha r}}
$$

where $w_{f}=882.9 \mathrm{~N}$ and $w_{r}=902.5 \mathrm{~N}$ are the static weights on the front and rear tires. It can be found that the understeer gradient $K$ is $0.62 \mathrm{deg} / \mathrm{g}$, which means that the car is slightly understeer. It must be 


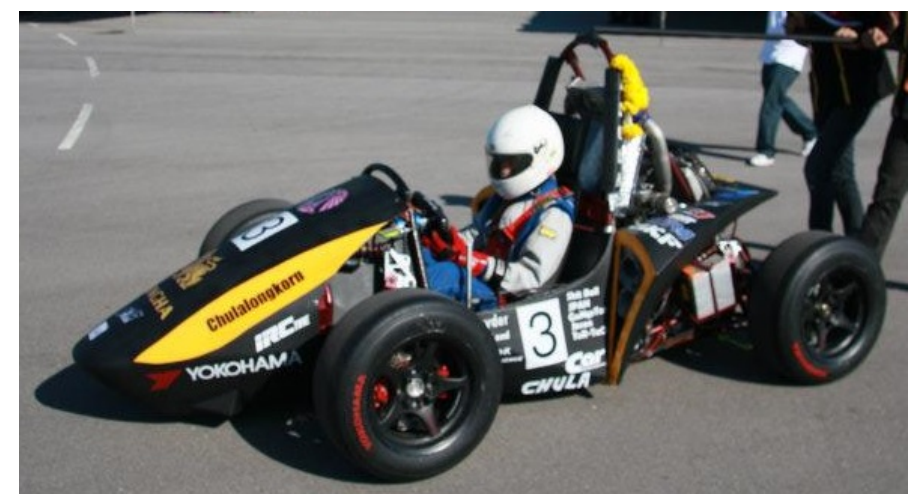

Fig. 10. Chulalongkorn 2012 Formula SAE car.

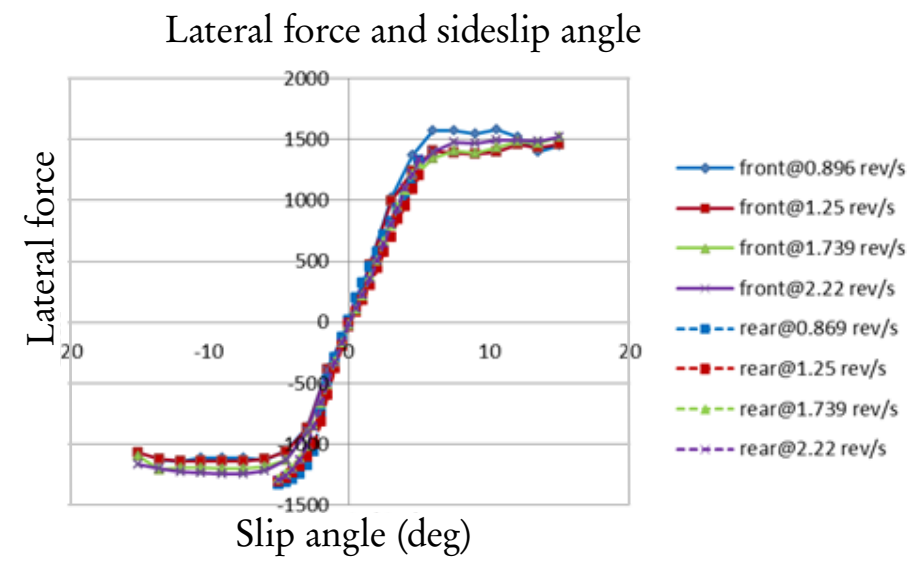

Fig. 11. Lateral force as a function of slip angle for the front and the rear tires

noted that this prediction of understeer behavior should be quite accurate at low lateral accelerations (low lateral forces).

\section{Experimental Results}

Skidpad tests were used to test the dynamic behaviour of the Formula SAE car. In this test, a skidpad with a radius of 10.6 meters was used. An accelerometer and a steering rack stroke sensor were used to record data while the driver was slowly increasing the speed of the car. The vehicle used in this test was the 2012 Chulalongkorn University Formula SAE car shown in Fig. 10.

Figure 12 shows the results of the skidpad experiments. In this figure, two driving experiments are shown. The results from the HIL simulations are shown as diamonds in the figure. Note that for the HIL simulations, the actual front wheel with suspension and steering components were used, while a nonlinear tire force model based on Fig. 11 was used for the rear tire. For comparisons, two non-HIL simulations using the same single track model with a linear and a nonlinear tire models are also shown. Note that for the steady-state skidpad tests, skidpad results for the non-HIL simulation can be directly calculated without requiring any numerical simulation. Assuming a small steering angle, skidpad results for the non-HIL simulation with linear tire model (dashed line) can be calculated using Eq. (3).

$$
\delta=\frac{L}{R}+K a_{y}
$$




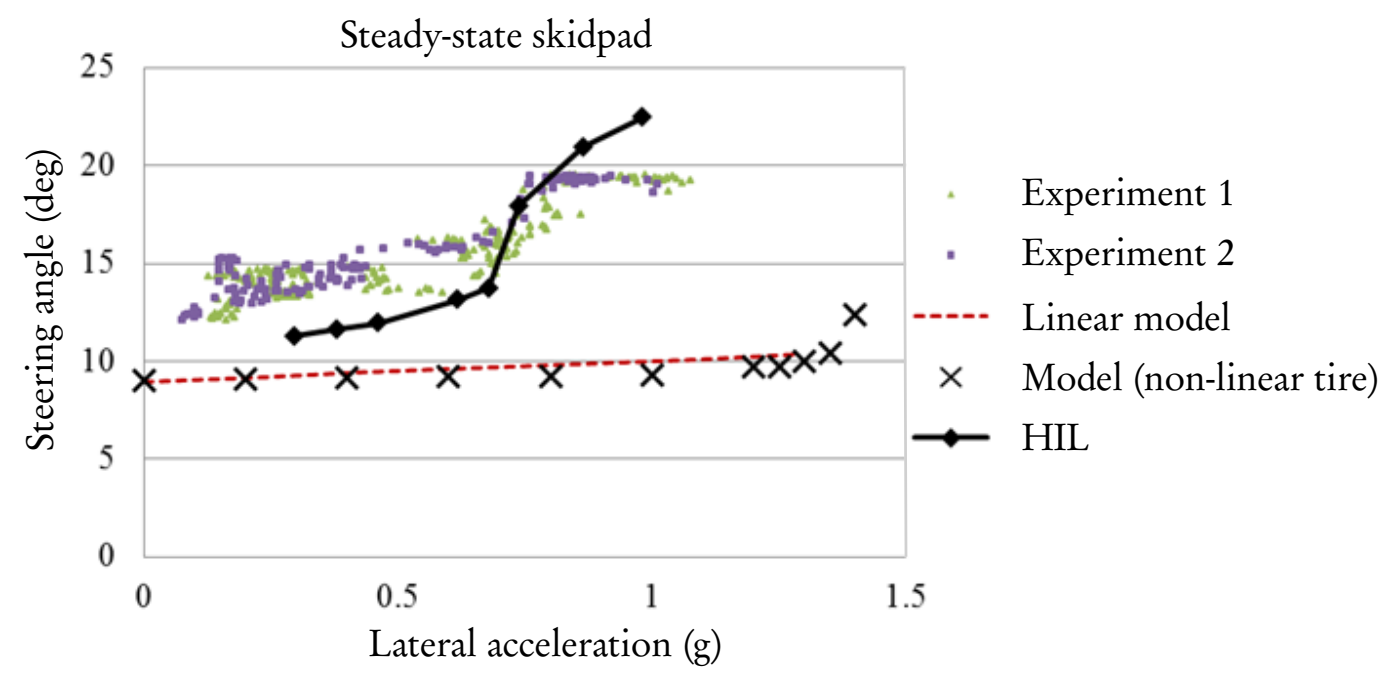

Fig. 12. Skidpad experimental results compared to HIL simulation and models with linear/nonlinear tire model.

where $\delta$ is the steering angle; $L$ is the wheel base of the car $(L=a+b) ; R$ is the turning radius; $K$ is the understeer gradient; and $a_{y}$ is the lateral acceleration [24]. Similarly, the non-HIL results with a nonlinear tire model (' $x$ ' markers) can also be calculated by rewrite Eq. (3) as

$$
\delta=\frac{L}{R}+\alpha_{f}-\alpha_{r}
$$

Given a lateral acceleration, the required lateral forces may be calculated as

$$
\begin{aligned}
F_{y_{w} f} & \approx m a_{y} b / L \\
F_{y r} & \approx m a_{y} a / L
\end{aligned}
$$

Using Fig. 11, the required slip angles $\left(\alpha_{f}\right.$ and $\left.\alpha_{r}\right)$ can be found and used in Eq. (4).

From Fig. 12, it can be seen that the HIL simulation results are better at predicting the actual experiments compared to the non-HIL results. If we consider the maximum lateral acceleration, it can be seen that the maximum lateral acceleration predicted by the HIL simulation is closer to the value obtained by the experiments which is around $1 \mathrm{~g}$. On the other hand, it is around $1.4 \mathrm{~g}$ for the non-HIL simulation with a nonlinear tire model. It can be clearly seen from Fig. 11 that the lateral acceleration limit around 1.3-1.5g may be expected since the lateral force becomes saturated at 30\% to $50 \%$ more than the static weights on that tires. Note that when the nonlinear force data in Fig. 11 is used, the results will not deviate much from the linear force model (dashed line) as shown in Fig. 12 unless the forces are near saturation. Although all simulation results are somewhat linear and have somewhat the same upward trends below $0.6 \mathrm{~g}$, the experimental values quickly increase (becoming more understeer) around $0.7 \mathrm{~g}$, which is also predicted by the HIL simulation.

Apart from various measurement errors, which should be minimized, such as steering angle and acceleration measurement errors, there are some factors that the non-HIL simulation cannot capture. Three important factors are the deformation of the components, camber change due to body roll, and the effect of lateral weight transfer. In the experiment, side forces cause deformation of the suspension and the steering components which may increase or decrease the steering angle. The other two factors are caused by the lateral acceleration. At a larger value of lateral acceleration, the vehicle body would slightly roll and generate lateral load transfer. Camber change causes by the body roll and the lateral load transfer would change the ability of the tire to generate lateral force at the same slip angle. As a result, the driver must steer to compensate. These factors caused a large different in steering angles 
when compared to the non-HIL simulations. The HIL simulation, however, attempted to capture the first factor. From Fig. 12, it can be seen that the HIL simulation results are better at predicting the actual experiments compared to the non-HIL results. From the figure, it may be suggested that the first factor is the most important.

The step steering test was another type of test performed in this work. In this case, a step steering of 7 degrees at the wheel with a rate of $70 \mathrm{deg} / \mathrm{s}$ was used. The step was at 1.5 seconds and the car speed was $25 \mathrm{~km} / \mathrm{h}$. The lateral acceleration $\left(a_{y}\right)$ and the sideslip angle $(\beta)$ were measured at the rate of 100 $\mathrm{Hz}$ using a GPS/GLONASS based Vbox 3i SL measurement system with an Inertial Measurement Unit (IMU). Note that another version of the Formula SAE car shown in Fig. 13 was used in this test with the following parameters: $m=428 \mathrm{~kg}, a=0.87 \mathrm{~m}, b=0.72 \mathrm{~m}, I_{z}=175 \mathrm{~kg} / \mathrm{m}^{2}, C_{\alpha f}=485 \mathrm{~N} / \mathrm{deg}$, and $C_{\alpha r}=585 \mathrm{~N} / \mathrm{deg}$. The step steering responses are shown in Fig. 14 and Fig. 15. In both figures, the gray lines are the experimental results and the black lines are the results from HIL simulations. The non-HIL simulation results are shown as dashed lines in the figures. The non-HIL results were simulated based on Eq. (1), assuming that the car speed $\left(V_{x}\right)$ is constant. The lateral forces were modeled linearly with $F_{y_{w} f}=C_{\alpha f} \alpha_{f}$, and $F_{y r}=C_{\alpha r} \alpha_{r}$. Nonetheless, it should be noted that if nonlinear tire force models were used the results would not be significantly different since the lateral forces were well within the linear region in this simulation.

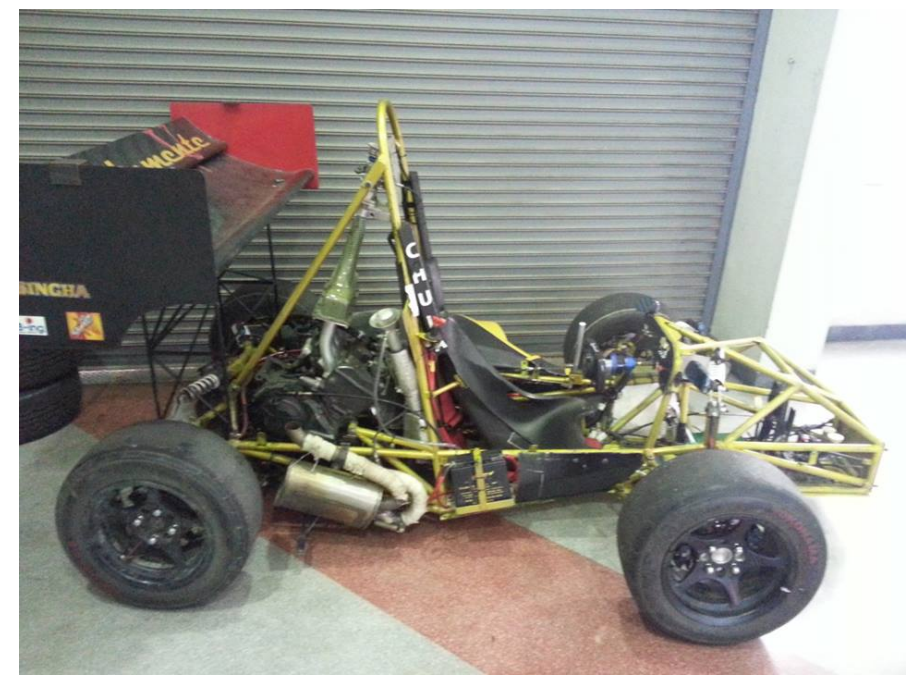

Fig. 13. Chulalongkorn 2013 Formula SAE car.

In terms of the step responses in Fig. 14 and Fig. 15, it is clear that the results from the HIL simulations match better with the real experiments when compared to the non-HIL simulations, especially for the lateral acceleration responses shown in Fig. 14. Furthermore, the steady-state values of the HIL simulation matches better with the results from the experiment. In particular, the steady-state value of the non-HIL simulation is accurate only for the sideslip value as shown in Fig. 15. It should be note that, in both figures, the responses of the linear bicycle model are faster compared to the others as seen by the steeper slopes at the beginning of the steps. This may be explained by the lack of dynamics of tire force generation. In particular, it takes time for the tire to deflect and develop side force. Only the HIL simulations can capture this because of the actual tire was used. For the sideslip angle response, although similar to the experiment, the HIL simulation cannot capture the undershooting at the beginning of the step response. Although, the causes of this is unclear, it may be suggested that it was caused by the difference in speed of side force generation between the front and the rear tire which was not capture by the HIL simulation since the force model of the rear tire has no dynamics. 
Lateral acceleration responses with step steering

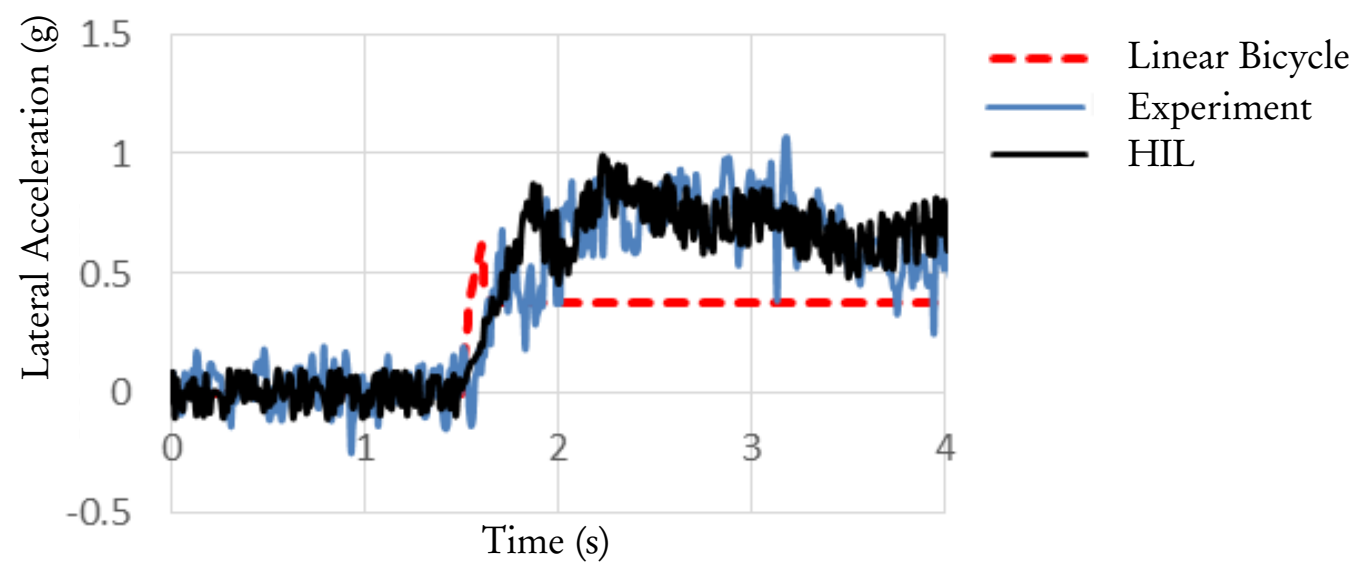

Fig. 14. Lateral acceleration responses (step steering).

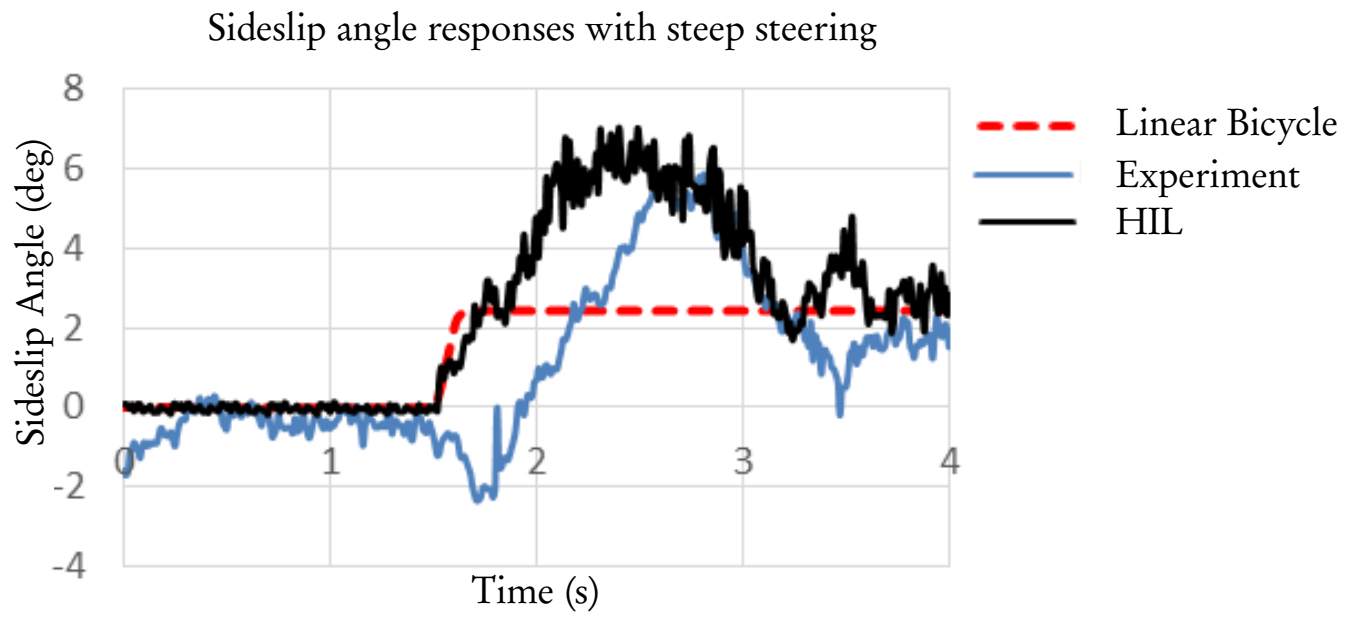

Fig. 15. Sideslip angle responses (step steering).

\section{Conclusion}

In this work, a newly developed HIL system with tire, suspension, and steering components is presented. In the literature, only HIL systems with separate components proposed in this work have been studied. Designs and configurations of the HIL system are shown. Comparisons of HIL simulations and real experiments using the skidpad and the step steering tests are given. It was found that the HIL simulation results are more accurate compared to the non-HIL simulations.

\section{Acknowledgement}

This research was a part of a project conducted at the Vehicle Dynamics Lab, Smart Mobility Research Center (SMRC), Department of Mechanical Engineering, Faculty of Chulalongkorn University. This project was partially funded by the Special Task Force for Activating Research (STAR), Ratchadaphiseksomphot Endowment Fund, Chulalongkorn University, the Chulalongkorn Academic Advancement 
into its 2nd Century Project, and the National Metal and Materials Technology Center (MTEC), Thailand, under the project "Development of Novel Evaluation Systems for Vehicle Dynamics, Driving, and Components."

\section{References}

[1] O. J. Gietelink, J. Ploeg, B. D. Schutter, and M. Verhaegen, "Development of a driver information and warning system with vehicle hardware-in-the-loop simulations," Mechatronics, vol. 19, no. 7, pp. 1091-1104, 2009.

[2] S. Hyun-Chul, K. Hong, and J. K. Hedrick, "Semi-active control of the macpherson suspension system: HIL simulations," in Proceedings of the 2000 IEEE International Conference on Control Applications, 25-27 September, 2000, Alaska, USA, 2000, pp. 982-987.

[3] W. E. Misselhorn, N. J. Theron, and P. S. Els, "Investigation of hardware-in-the-loop for use in suspension development," Vehicle System Dynamics, vol. 44, no. 1, pp. 65-81, 2006.

[4] R. Isermann, J. Schaffnit, and S. Sinsel, "Hardware-in-the-loop simulation for the design and testing of engine-control systems," Control Engineering Practice, vol. 7, no. 5, pp. 643-653, 1999.

[5] S. H. Lee, T. W. Park, K. H. Chung, K. H. Choi, K. K. Kim, and K. H. Moon, "Requirement-based testing of an automotive ECU considering the behavior of the vehicle," International Journal of Automotive Technology, vol. 12, no. 1, pp. 75-82, 2011.

[6] K. Enisz, D. Fodor, I. Szalay, and L. Kovacs, "Reconfigurable real-time hardware-in-the-loop environment for automotive electronic control unit testing and verification," IEEE Instrumentation $\mathcal{E}$ Measurement Magazine, vol. 17, no. 4, pp. 31-36, 2014.

[7] V. Rajaram and S. C. Subramanian, "Design and hardware-in-loop implementation of collision avoidance algorithms for heavy commercial road vehicles," Vehicle System Dynamics, vol. 54, no. 7, pp. 871-901, 2016.

[8] M. H. Lee, H. M. Lee, K. S. Lee, S. K. Ha, J. I. Bae, J. H. Park, H. G. Park, H. J. Choi, and H. H. Chun, "Development of a hardware in the loop simulation system for electric power steering in vehicles," International Journal of Automotive Technology, vol. 12, no. 5, pp. 733-744, 2011.

[9] W. Baek and B. Song, "Design and validation of a longitudinal velocity and distance controller via hardware-in-the-loop simulation," International Journal of Automotive Technology, vol. 10, no. 1, pp. 95-102, 2009.

[10] B. Ashok, S. D. Ashok, and C. R. Kumar, "An integrated pedal follower and torque based approach for electronic throttle control in a motorcycle engine," Engineering Journal, vol. 21, no. 1, pp. $63-$ 80, 2017.

[11] J. H. Song, J. X. Wang, H. B. Tang, X. J. Mao, and B. Zhuo, "Diesel hybrid electric vehicle hardware system," International Journal of Automotive Technology, vol. 10, no. 4, pp. 523-528, 2009.

[12] N. Noomwongs, H. Yoshida, M. Nagai, and S. Kawamata, "Development of hardware-in-the-loop simulator for analyzing vehicle handling and stability," in Dynamics and Design Conference, 6-9 August 2001, 2001.

[13] N. Noomwongs, H. Yoshida, M. Nagai, K. Kobayashi, and T. Yokoi, "Study on handling and stability using tire hardware-in-the-loop simulator," JSAE Review, vol. 24, no. 4, pp. 457-464, 2003.

[14] H. H. Sung, J. H. Seung, S. K. Hong, and I. L. Kyo, "Vehicle dynamic analysis and evaluation of continuously controlled semi-active suspensions using hardware-in-the-loop simulation," Vebicle System Dynamics, vol. 27, no. 5-6, pp. 423-434, 1997.

[15] A. Bertacchini, L. Tamagnini, and P. Pavan, "Force feedback in steer-by-wire systems: Architecture and experimental results," in Proceedings of IEEE International Symposium on Industrial Electronics, 9-12 July 2006, Quebec, Canada, 2006, pp. 3050-3055.

[16] L. Heidrich, B. Skyrokau, D. Savitski, V. Ivanov, K. Augsburg, and D. Wang, "Hardware-in-theloop test rig for integrated vehicle control systems," in 7th IFAC Symposium on Advances in Automotive Control, The International Federation of Automatic Control, Sep. 4-7, 2013, Tokyo, Japan, 2013. 
[17] D. C. Batterbee and N. D. Sims, "Hardware-in-the-loop simulation of magnetorheological dampers for vehicle suspension systems," in Proceedings of the Institution of Mechanical Engineers. Part I: Journal of Systems and Control Engineering, vol. 221, no. 2, 2007, pp. 265-278.

[18] K. Hudha, M. H. Zakaria, and N. Tamaldin, "Hardware in the loop simulation of active front wheel steering control for yaw disturbance rejection," Int. J. of Vehicle Safety, vol. 5, no. 4, pp. 356-373, 2011.

[19] S. Lapapong, V. Gupta, E. Callejas, and S. Brennan, "Fidelity of using scaled vehicles for chassis dynamic studies," Vehicle System Dynamics, vol. 47, no. 11, pp. 1401-1437, 2009.

[20] R. Chaichaowarata and W. Wannasuphoprasit, "Kinematics-based analytical solution for wheel slip angle estimation of a rwd vehicle with drift," Engineering Journal, vol. 20, no. 2, pp. 89-107, 2015.

[21] P. Boonchata, N. Noomwongs, and S. Chantranuwathana, "Development of tire-suspensionsteering hardware in the loop simulator for student formula car handling testing," in Proceedings of the 17th Asia-Pacific Automotive Engineering Conference, SAE Technical Paper 2013-01-0004, 2013.

[22] S. Sookchaiyaporn, N. Noomwongs, R. Chanchareon, W. Wannasuphoprasit, and S. Chantranuwathana, "Tire-suspension-steering hardware-in-the-loop for vehicle dynamics simulation," in Proceeding of the 15th Asia Pacific Automotive Engineering Conference, 26-28 October 2009, Hanoi, Vietnam, 2009.

[23] L. D. Novellis, A. Sorniotti, P. Gruber, L. Shead, V. Ivanov, and K. Hoepping, "Torque vectoring for electric vehicles with individually controlled motors: State-of-the-art and future developments," in EVS26 International Battery, Hybrid and Fuel Cell Electric Vehicle Symposium. 6-9 May 2012, California, USA, 2012.

[24] T. D. Gillespie, Fundamentals of Vehicle Dynamics, 1st ed. Society of Automotive Engineers Inc., 2014.

[25] A. L. Svenson, P. A. Grygier, M. M. Kamel Salaani, and G. J. Heydinger, "Validation of hardware in the loop (HIL) simulation for use in heavy truck stability control system effectiveness research," in Proceedings of the 21st (ESV) International Technical Conference on the Enhanced Safety of Vebicles, 15-18 June 2009, Stuttgart, Germany, Paper No. 09-0189, 2009. 